\title{
Homebirth Advocacy on the Internet
}

\author{
Shira Segal \\ Indiana University, USA
}

\begin{abstract}
On-line depictions of the maternal and birthing body in the familial and communal context aims to destigmatize the "natural" (i.e., the non-intervened) body by putting birth - and representations of birth - back into women's hands and homes. The many rich and diverse representations of amateur homebirth home movies on the Internet are part of an on-going visual effort to make and exhibit visual documents of and about the maternal, birthing body that upholds the (w)holistic model of childbirth and thus furthering the emotional, visual, and political stakes of the homebirth movement both on-line and in real life. As part of the rich history of alternative representations of childbirth - and alternative birth practices such as homebirth - that reworks the traditionally taboo subject of childbirth with the said goal of empowering women and their families, contemporary online representations of homebirth are politically, corporeally, and emotionally invested.
\end{abstract}

[Key words: Internet, Childbirth, Visual Culture / Video, Gender, YouTube]

How do amateur videos of homebirth on-line invite us to re-examine autobiography, the everyday and diary film/video-making practices in regards to art, the body, and the personal-political-medical struggles of the contemporary homebirth movement on-line? Much like experimental homebirth films that can be couched within larger discourses of second wave feminism in the United States as well as the holistic health and back-to-earth movements of the 1960s and 1970s, these on-line amateur depictions continue the work of destigmatizing the "natural" (i.e., the non-intervened) procreative body in our society particularly in their attempt to put birth and representations of birth back into women's hands and homes - and then, importantly, out again into the public visual sphere of the Internet. Concerning the larger efforts of the homebirth movement to empower women and their families, both experimental and amateur homebirth films and videos share certain elements that are also popular in self-circulated midwife films such as the uptake of the everyday, the re-centering of the maternal subjective, and an emphasis on the communal and the collaborative. As part of the rich history of alternative representations of childbirth - and alternative birth practices such as homebirth - that reworks the traditionally taboo subject of childbirth with the said goal of empowering women and their families, contemporary on-line representations of homebirth are politically, corporeally, and emotionally invested.

In the case of experimental homebirth films, the recurring filmic treatment of the nature of birth is to allow the footage to stand relatively on its own, that is, unedited and in its entirety. The restrictions placed on such imagery generally stem from technical and practical factors - the duration of a film roll, the amount of available light, etc. In these cases, the camera is frequently the birthing family's friend and guest, witness and at the same time, collaborator. The spirit and philosophy of homebirth, one that is characterized by supporting and enabling the laboring woman's autonomy and empowerment in the context of community and family, gives way to a collaborative vision. The film becomes another way of honoring, listening, and responding appropriately to women's bodies and births, not only by documenting an intervention-free birth in a loving

Rupkatha Journal on Interdisciplinary Studies in Humanities

Volume 2, Number 1, Special Issue, Visual Arts

URL of the Issue: http://rupkatha.com/rupkathav2n1.php

PDF URL of the article:

http://rupkatha.com/V2/n1/HomebirthAdvocacyonthelnternet.pdf

(C) www.rupkatha.com 
and supportive setting but also by gifting that film to the family. The film can then be integrated into family birthday rituals and the family's (visual) story of itself in meaningful ways. Thus, the incredible physical/emotional transformation into parenthood that the process of birth embodies dictates the form (and potentially the reception) of the film - in line with homebirth philosophy, the film listens and responds to the subject, and is perhaps integrate-able into the family's understanding of itself.

The domestic images of childbirth by the amateur home movie maker who posts their videos on-line contrasts with these experimental homebirth films in a few ways, not only in terms of circulation and exhibition but in aesthetics as well. Although avant-garde filmmakers like Stan Brakhage $(1933=2003)$ and others have termed their work in the tradition and in the heart of the amateur - and distinctions between "artist" and "amateur" have been historically blurred by filmmaking practices that incorporate and rely on both - I argue here that the amateur film- or video-makers of my study can perhaps best be understood as a hobby-ists in the sense that they are not attempting to live off the proceeds of their visual work. Not purporting to be "art," and generally not dictated by form, I situate contemporary home-movies on the subject of childbirth as primarily subject-driven. Technology is certainly a significant contributing factor in this trend - the time constraint-free quality of analog and now digital video in addition to the computer-based and user-friendly editing software like Final Cut Pro and Adobe Premier, not to mention the economically viable choice to shoot on video rather than film, makes home-movie making practices less married in a sense to the form itself - and, as a result, more malleable in their use; perhaps because of this, hospitals may prohibit expecting parents from documenting their birthing experiences for fear that such videos may be used as evidence in potential malpractices lawsuits against the doctor or hospital.

Additionally, websites like YouTube (launched in 2005), the Internet's most used video-sharing tool, (along with GoogleVideo, Viddler and VideoWebTown) solve questions of exhibition and distribution so that anyone with a web-cam equipped computer or even a mobile phone can publicize their footage to the public at large, although it should be noted that YouTube currently requires viewers to sign-in and validate their birth date in order to access most video images of childbirth or other subjects deemed "inappropriate" by the YouTube community. Regardless, the wide-ranging variety of childbirth videos on the Internet is astounding, and there have even been efforts to collect and catalog them by websites like birthvideos.tk (alias pregnancyvideos.googlepages.com). Similar cataloguing efforts of birth imagery on-line have been applied to still photography, as seen by Stephanie Soderblom's Birth Story Diaries, sponsored by Nurturing Hearts Birth Services of Arizona, which "shows the reality of childbirth...the beautiful miracle it can be, and the difficulties it might present" (birthdiaries.com, accessed October 2009).

Indeed, still and moving imagery on the Internet surrounding the procreative and newborn bodies can be politically and personally invested, and frequently an aura of organization and desire for political and social change is palatable in these images. This is particularly true in homebirth videos that attempt to alter the atmosphere of fear surrounding birth and replace it with a culture of confidence by encouraging women to birth either on their own - best illustrated by Laura Shanley's videos of unassisted childbirth on her website BornFree! (unassistedchildbirth.com) - or with a homebirth midwife and/or a 
doula. For instance, the "Free Birth Videos" on birthvideos.tk1 are offered as a visual resource and response against fictional depictions of childbirth - "Because we have found TV births to be so unrealistic and even scary to watch, we urge people to watch real birth videos found here, captured on a simple camcorder and usually not heavily edited or dramatized. In a homemade birth video you will see what birth is really like" ("About Us," birthvideos.tk). The Internet as a location is argued to enable a virtual community gathering that fosters education, knowledge, and normalcy of birth. The website prominently features homebirth, waterbirth, and unassisted childbirth.

To avoid an inclusion of heavily medicated or highly intervened births such as Cesarean operations, birthvideos.tk lumps hospital and birth center births into one category, and frames many of the videos of this section within discourses that critique intervention - depictions of painful births and episiotomies, internal fetal monitors, and delivery via vacuum suction cups and forceps contrast with drug-free and "natural" births in the hospital and birth center setting that features various postures and methods (like hypnotherapy techniques for deep relaxation), family participation, and a general normalization of the birthing process. In fact, the presence of breech positioned and/or twin babies successfully delivered vaginally as well as the inclusion of vaginal births after Cesarean (video \#74 even includes a link to vbac.com, an anti-Cesarean website that, as a "woman-centered, evidenced based, resource" outlines the dangers of abdominal delivery) makes the "hospital and birth center" category of the website particularly charged - videos that triumphantly depict vaginal birth speak against institutional powers and the general medical sentiment (and resulting hospital policies in some cases) that previous Cesarean mothers or breech positioned babies have mandatory Cesarean operated births. Considering the current climate of anti-VBAC (vaginal birth after Cesarean) birth policies along with Cesarean section rates that exceed $30 \%$ of all hospital births in the United States that are driven by fears of litigation, the vaginal births on this website are triumphant, personal, and political.

The website's general anti-intervention sentiment that frames childbirth as "natural" and normal (and various levels of medical intervention as potentially disruptive and perhaps even harmful to both the mother and baby) is at its peak in videos that feature homebirth-hospital transfers that remain autonomous and, importantly, out of the supine position in which the mother labors on her back with her feet in stirrups (a convenient position for the birthing attendant, but a detrimental one for the birthing process). For instance, the description for video \#124 in the hospital and birth center category details a preference for homebirth as well as the possibility of various birthing positions despite the medical setting in which the birth ended up, "After laboring at home for several days and hoping for a homebirth, the mother finally needs to transfer to the hospital. She pushes using a 'tug rope,' or pulling on a rope or towel for leverage. She at last delivers on her hands and knees" (birthvideos.tk). Various birth positions can be seen across the website's range of visual categories, along with a good dose of defiance towards medical authority/knowledge, as seen in the following descriptions from the homebirth category,

After her doctor told her she should have a c-section, a mother chooses to have a home waterbirth of her breech baby with a midwife instead. She delivered a healthy baby girl vaginally. (video \#72, [sic]) 
After her first baby was induced in the hospital, a mother has an unassisted birth at home for her second. (video \#62)

Her doctor told her that her first baby was too big and performed a csection. Now for her second baby, a mother has a successful homebirth. Her second homebirth baby was a pound bigger than her first c-section baby! (video \#50, [sic])

Additionally, a significant trend across categories is the favoring of family involvement, best illustrated in the homebirth category when siblings to those being born actively participate in the birth. One such example describes the following scenario,

For her tenth child, a boy weighing $10 \mathrm{lbs}$. 3 oz., a woman has a home waterbirth unassisted. Her husband holds her hand while their 13-year-old daughter filmed the birth, and their 15-year-old daughter helped deliver the baby. The entire labor was only about an hour. (video \#53)

Overall, a general normalization of the birth process pervades the stories and images of birth on birthvideos.tk. For instance, also from the homebirth category, the following description instructs the viewer on what to expect and proposes a particular attitude towards the various aspects of the birth process,

In this wonderfully intimate look at a peaceful childbirth, a couple has a homebirth in their apartment of their first child. As is common for first babies, the pushing stage lasts quite a while--for this mother four hours. The baby's head crowns for about an hour before finally delivering, which is normal and actually beneficial, because it helps to stretch the skin and prevent tearing. Lots of meconium, or the baby's first bowel movement, was present at birth. (video \#70)

Even the visual category of "unique birth videos" and its division into three subcategories encourages an attitude of confidence around birthing women and their babies: 1) "unique babies" refers to how babies are born rather than their intrinsic qualities, such as babies born breech (feet or buttocks first), in the posterior position (rather than facing the mother's spine which makes the 90 degree turn around the mother's pubic bone an easier journey for the baby's head, posterior babies face the mother's pubic bone), or with the amniotic sac still intact; 2) "unique birthing positions" includes examples of women who birth while standing, hammock-reclining or while in the great outdoors (a backyard), and 3) "special mothers" like a woman with multiple sclerosis who has an unassisted birth, a teenage mother who opts for a waterbirth or a circumcised mother in West Africa (although in the final instance, the website makes it clear that they do not condone female circumcision, "Female circumcision/genital mutilation often causes complications in childbirth and creates an increase risk of fetal death" [sic]).

In general, birthvideos.tk wears their stance against intervention-based birth practices on their sleeve along with a good does of skepticism towards medical thinking that restricts how and when women birth. For instance, video \#26 in the hospital and birth center states "A nine-pound overdue baby with a 14inch head is delivered drug-free in a hospital. The mother delivers on her stomach," thus defying the myth that women should not be allowed to go past 
their due date and the myth's accompanying rationale that women's pelvises are too small or their babies' heads too big; whereas a diagnosis of Cephalopelvic Disproportion [CPD] would appear to indicate severe malnutrition resulting in rickets or a severe pelvic injury, such a diagnosis is often given to women who are deemed as failing to progress in labor, resulting in Cesarean operation, or according to the International Cesarean Awareness Network, may be entered in a woman's medical records before she has a chance to labor.2 Similar examples of triumphant vaginal deliveries both in and out of the hospital setting are featured as birthvideos.tk's video of the week; the heading for one such birth video of the week describes a successful vaginal birth in the hospital, "Mommy gives birth to her fourth child in the hospital without drugs, a huge $10 \mathrm{lbs}$. $2 \mathrm{oz}$. little girl. It was her most difficult birth yet, but she was determined to do it naturally" (November 2, 2009). Indeed, while the website's political message is at its peak in its homebirth imagery, the preference for homebirth and its womancentered practices that are rooted in a culture of confidence surrounding women's ability to birth is implied throughout the website.

However, the political potential of an increased visibility of the maternal birthing body on-line is arguably stunted by the increasing commercialization of homebirth and its images. For instance, the "Free Birth Videos" on birthvideos.tk come with the cost of navigating their website that is cluttered with third party advertising that tout things related to pregnancy (baby sleeping bags) and not (car insurance, stool softeners, nail salons services) - not to mention the announcement that they are considering selling the website and open to offers (September 2009). The premise of birthvideos.tk that the depiction of "real" birth videos, unlike the unrealistic fictional depictions of (hospital) births on television, are the best tools for education, inspiration, community building and empowerment arguably gets lost amidst their current web layout and outside advertising efforts - what appears to be headlines for dividing birth videos into various categories are actually Google Ads, which are shortly followed by a "sponsored links" subheading and advertising sidebar links. Additionally, the political relevance of the website is potentially undermined by the inclusion of particular links on birthvideos.tk's homepage that are seemingly unconnected to birth - as "original" texts of the website (rather than additional advertisements that are externally-generated), alliances include blogs like Divorce, Gosselin Style that tediously follows and comments on the break up of celebrity couple Kate and Jon Gosselin (stars of The Learning Channel's reality television show Jon \& Kate Plus Eight that features the couple and their eight children) and $165 \mathrm{~K}$ in Debt (apparently authored by the same attorney as the Gosselin blog). To be fair, birth-related (and homebirth friendly) links such as Pregnancy Today's birthstories.com, the Hip Chick's Pregnancy Guide and Mothering Magazine's Birth Forum far outweigh these unrelated-to-birth instances, yet even "natural childbirth" and "natural families" websites that are politicized as homebirth advocacy organizations similarly suffer from product-driven sponsorship that targets expecting parents.

Another example of the tension between profit making and virtual community organizing on-line is highlighted in Ricki Lake and Abby Epstein's website mybestbirth.com (which only recently appears in birthvideos.tk's new "links" section [October 2009]; many birth videos on these two websites are echoed or shared by each other). Framed as "Ricki Lake's community for people to share experiences and options about pregnancy, natural birth and holistic 
parenting" and boasting 7,476 members and 74 groups that range from "Baby Sign Language," "Extended Breastfeeding" and "Baby wearing" to "LGBT Families and Families-To-Be" (Lesbian, Gay, Bisexual, Transgender), "Genital Integrity" and "VBAC Moms" (Vaginal Birth After Cesarean) (as of September 2009; membership increased to 7,893 by November 6,2009 ), the website also promotes their new book Your Best Birth - Know All Your Options, Discover the Natural Choices, and Take Back the Birth Experience (2009) and reinforces the popularity of their recent homebirth advocacy documentary The Business of Being Born (2008). Unsurprisingly, mybestbirth.com offers a good share of birthrelated consumer products as well, from cord blood banking (stork.md), natural fertility management (naturallyforher.com), biodegradable and flushable diapers (gdiapers.com), organic and vegetarian baby food and other non-toxic products (sproutbaby.com) - the consumer base for these products appears to be middle to upper class mothers and families.

The marketability of homebirth extends beyond birth-related items and topics, however, illustrated by Edu Cuèllar's tasteful 2009 Flex mattress commercial that aired on television in Spain in April 2009, featuring a Barcelona couple discussing and then giving birth in their bed at home and in the company of their son and a midwife, followed by the tag-line "Your bed, the most important place in the world" (2009). Indeed, we've come a long way from what was the radical integration of Lucille Ball's real-life pregnancy in her fictional character on the I Love Lucy show in 1952 - especially given the many restrictions placed on such representations, such as the inability to say the word "pregnant" on broadcast television - but we have not shed the marketability of the pregnant subject (Ball's pregnant appearance on television not only coincided with but also bolstered the burgeoning market of the maternity clothing industry3).

Today, amidst the plethora of cable television reality shows that feature childbirth (including The Discovery Health Channel's Deliver Me, The Oxygen Network's Birth Stories, and MTV's 16 and Pregnant inspired by Jason Reitman's fictional teen pregnancy film Juno [2007]), it is only The Learning Channel's Baby Story and Bringing Home Baby that occasionally attempt to feature homebirth. These stand out as radical (and lonely) instances of a potentially nonpathologized procreative body on television, particularly given the hospital-heavy emphasis across the board when it comes to the birthing body at large in our culture. For instance, the emphasis on intervention (from amniocentesis and induction to surgical delivery) as necessary and normal steps for a successful birth on the high risk pregnancies featured in Discovery Health's Deliver Me reinforces the already-widespread technological perspective that situates pregnancy as a disease to be managed and fixed by medical personnel, so much so that the rare (and seemingly successful) "natural" birth on this show (youtube.com/watch?v=SOqc7rRAld0) still includes a transfer to the hospital, the breaking of the bag of waters and a final pushing stage in the partial lithotomy or supine position (falsely claimed by the doctor to be the best "angle" for mother and the baby, despite evidence that this is in fact the worst position for them both). Indeed, the rare instance of homebirth on Cable television starkly contrasts with the bulk of pregnant reality television shows that function to contain the maternal body particularly when it is birthing, evidenced by the predictable transformation of birth into a controllable (i.e., intervened) procedure or medical event. 
If anything, Cable television's sporadic attempt to feature homebirth (which wanes more than it waxes, much like these programs' spotty inclusion of gay or lesbian parents) and the commercialization of homebirth reflects the trendy marketability of the pregnant body itself. From belly advertising (in 20052006 a handful of women auctioned their pregnant abdomens as advertising space to the highest bidder on eBay, resulting in company sponsored pregnancies and births4) to pregnant women's appearances in advertising for family sports cars, maternity lingerie, nonalcoholic beer, and even vodka, the pregnant body has a range of visual uses in our culture (including pornography). The pregnant body carries visual agency beyond product-driven images - Daniel Edwards' sculpture of a nude Britney Spears laboring on all fours on a bearskinned rug (despite her actual Cesarean section) titled "Monument to Pro-Life: The Birth of Sean Preston" (2006, caplakesting.com/danedwards/index.htm) is perhaps an extreme example of pro-life discourses that show up (albeit less blatantly) in films like Adrienne Shelley's Waitress, Judd Apatow's Knocked Up, and Reitman's Juno (all 2007). In contrast to the multiple uses of the pregnant body and the abundance of medical childbirth practices depicted in mainstream media, visual instances of homebirth advocacy in our visual culture take on a valuable role. Often framed in terms of maternal subjectivity and bodily rights, homebirth advocacy documentaries like Gilles de Maistre's Le Premier Cri (2007) and Debra Pascali-Bonaro's Orgasmic Birth - The Best Kept Secret (2008) directly address the benefits of non-intervention childbirth practices, as does the on-line homebirth video competition for Birth Matters Virginia, which describes evidence-based maternity care as "using the best available research on the safety and effectiveness of specific practices to help guide maternity care decisions and to facilitate optimal outcomes in mothers and newborns" (birthmattersva.org, accessed October 20, 2009).

Non-professional and professional homebirth videos on-line like those in the birthmattersva.org video competition demonstrate an important visual and political alternative to mainstream depictions of pregnancy, childbirth practices, and attitudes towards women and the birthing process. In fact, the prevalence of on-line homebirth videos in which birthing women and their families tout the benefits of non-intervention in their depictions of doula and midwife attended births makes one forget that birth outside of the hospital still remains a fringe birthing practice in the United States. The homebirth, free-birth, anti-Cesarean and anti-intervention advocacy birth videos that preach to the choir, so to speak, already touch on the following list of potential topics for those wishing to participate in the Birth Matters Virginia video competition,

1. The role of doulas and labor assistants in childbirth

2. Appropriate and inappropriate uses of various interventions (pitocin, epidural, episiotomy, breaking of waters, forceps, vacuum extraction, etc.) [sic]

3. The advantages of child birth education classes or explorations of different methods [sic]

4. The reasons behind the rising c-section rate in the United States [sic]

5. The risks and rewards of VBAC [Vaginal Birth After Cesarean]

6. The advantages and/or rewards of unmedicated birth

7. Pros and cons of different birthing location (hospital, birthing center, home)

8. How to facilitate postpartum bonding between mother and baby 
9. Common arguments given for interventions; how and when to rebut them

10. Effective substitutes for common interventions (e.g. how to induce labor through natural methods, how to manage pain without an epidural, etc.)

11. The "domino effect" of interventions (i.e. how interventions increase the chances of subsequent interventions or c-section). [sic]

(birthmattersva.org/videocontest.htm/\#topics, accessed on October 20, 2009)

Many of the 2009 finalists do indeed address these issues, from Ragan Cohen's Prevent Cesarean Surgery (first prize, \$1000) and Laura Alvarez's The Nature of Natural Birth (second prize, \$500) to Julie Clevidence's Do You Doula?, Marla Yeatts's Doulas Make a Difference and Rachel Zucker's Home Birth/Water Birth of Judah Darwin. However, many of these videos rely on the "slideshow" of still photographs and in many cases seem to fall beyond the home movie category; in some cases the videos are interview-heavy or do not actually show a birth, either in moving or still images.

For instance, Kip Hewitt's Natural Born Babies, one of the finalists in the Birth Matters Virginia video competition, interviews mothers and couples of Orange County's South Coast MidWifery Birth Center who talk about how empowering it was to go through a natural birth. The video lacks birth imagery but is not short of birth stories and moving images of the babies and children whose births are described. Truncating and intercutting these interviews (along with expressive close-ups of the babies and children being held or smiling into the camera) functions as an elongated but sensitive advertisement for the accredited birth center which undoubtedly profits from these publicized yet personal accounts of positive birth experiences either at the center or at home (with the birth center's midwives) in contrast with the same couples' stories of negative hospital birth experiences and detrimental medical attitudes and practices towards birthing women's bodies. For instance, one participant explains that

I didn't want any intervention, I just wanted to be able to have the baby and be supported, and I thought the hospital was there to just be a backup measure in case anything went wrong. And then I started to find out that the hospital is not a backup measure, the hospital is there to get in the way. And really what you need if you just want someone to support you and make sure everything is safe is you need a midwife. (Wende Zomnir, founder of Urban Decay Cosmetics)

Similarly, one woman explains, "I was completely oblivious of the effects of pitocin and epidural that that has on the baby. I didn't discover that until after she came ... The recovery was amazingly easier than the first time. I didn't take any medication and I was fine the next day - I couldn't believe it" (Karen Eaton, homemaker) while another happily recalls her out-of-the-hospital birth that "We had guests over within the hour, I was able to be social, I ate a big meal, I was nursing my baby - I felt alive, I felt great" (Dr. Kaisa Coppola, chiropractor). Another participant dispels the stereotypes associated with women who seek out natural childbirth, 
The delivery was much easier than I thought. It is tolerable for a normal woman. You don't have to be - I don't want to insult anybody [laughing] but - you don't have to be $100 \%$ a vegetarian, and strong, and "earth mama" type of person. You can just be a normal girl and still make it, so it's pretty cool. (Kim Binford, church minister)

As seen from these examples, Natural Born Babies on the whole features professionals (an acupuncturist and chiropractor couple, a yoga instructor, an anesthesiologist and nurse practitioner couple, a church minister, a cardiologist and E.R. physician couple, among others of middle to upper class economic standing) and is visually polished. Against a white backdrop and consistently over-lit, which produces a crisp and professional image, the participants speak directly and candidly to the camera with Geroge Gershwin's "Rhapsody in Blue" as a background soundscape. The concluding statement wraps up the 17 minute video with what might easily be considered a slogan not only for that particular birth center but also for an economically privileged perspective on birth, "You give birth the way you live life. If you live life in your own way, then you might want to have a birth in your own way" (Zomnir).

Although Hewitt's Natural Born Babies clearly falls beyond the home movie category that is typically seen in the amateur (home)birth videos posted to birthvideos.tk and mybestbirth.com, Hewitt's video (along with a handful of other video finalists for the Birth Matters Virginia video competition) works directly with - and helps establish a professionally-endorsed branch of - visual discourses of "natural birth" advocacy that is then available on YouTube and other videosharing sites. Birth Matters Virginia judge Dr. Sarah Buckley (author of Gentle Birth, Gentle Parenting - A Doctor's Guide to Natural Childbirth and Early Parenting Choices [2008]) calls the video "A powerful argument for natural birth" and comments, "What I loved about this video was the clarity in all ways: visually, informationally, structurally and editorially." Another judge (unnamed) exclaims, "This could be a commercial for our mission statement! It's everything l'd want people to know about evidence-based care and choice in birth" (birthmattersva.org/videocontest.html, accessed on November 16, 2009).

Hewitt's efforts to alter the atmosphere of fear surrounding birth and supplanting it instead with a culture of confidence taps into the homebirth "spirit" while taking away the threatening and/or more graphic (i.e., visual) aspects of birth. This contrasts with much of the hand-held home-made imagery on-line of the unassisted and homebirth movements that is grainy, undirected, unedited, under-lit, unsteady, and for the most part visually (or at the very least, aurally) explicit. For instance, video \#69 in birthvideos.tk's homebirth category offers a clumsily hand-held depiction of a seventeen year old giving birth on her hands and knees, assisted by her mother and grandmother and in the company of at least one younger sibling. One could say that the birth is, in Robin Blaetz's terms, heard "in its lived fullness" $(1992,18)$ even though the majority of the footage is visually undecipherable due to the low lighting conditions. The visual clarity like that in video \#47 in the homebirth category ("After a disappointing hospital birth, a couple chooses their Jacuzzi outside in their backyard for the birth of their second child. Mother sits in the father's lap and delivers the baby into her own hands" - what this description leaves out is the intermittent laughter and ease of the laboring mother) relies on the off-chance of a steady camera person (or the 
rare use of a tripod) and a birth that occurs during the day - and, in this case, outdoors and with good weather.

In the spirit of empowering and inspiring women to have "normal" and "natural" birthing experiences in familial and communal contexts, most of the homebirth imagery on birthvideos.tk arguably romanticizes and desensitizes birth simultaneously. Birth is depicted in such a way that on the one hand builds up the birthing process as frequently magical, meaningful and worth documenting while on the other hand undercuts the mythos of birth by showing the physical reality and frequent struggle of it for women and on their bodies. In fact, these "hard" elements of birth and labor function in and of themselves as another layer of birth's spectacle even as the traditional spectacle of birth (i.e., the stretching maternal body as well as the newly emerged newborn body) is undermined by the efforts made to normalize the birth process and maternal/newborn bodies in these videos. As a result, the "normal" birthing body may be depicted as a site of struggle, abundance and strength as well as magic, uncertainty, sexuality and, perhaps at times, fear.

These videos frequently include moments of uncertainty and perhaps alarm for the unprepared viewer, such as the quick staining and spreading of blood in the tub's waters once the baby in video \#53 from birthvideos.tk's homebirth category (mentioned earlier, of a woman water-birthing her tenth child, clumsily documented by her thirteen year old daughter) emerges, or the baby's seemingly unresponsiveness (accompanied by diegetic music playing in the room - at the moment of would-be panic at the baby's lethargy, the song happens to be Paul Simon and Art Garfunkel's "Hazy Shade of Winter"). Another potentially disturbing video from the homebirth category is video \#13 in which the new mother appears to be in shock after the birth of her baby. However, it is not birth itself that appears scary in these videos but rather the conditions and consequences of birth on maternal, newborn and familial bodies. Ultimately, amateur homebirth films and videos are best understood within homebirth advocacy efforts to visually combat the atmosphere of fear surrounding birth with a culture of confidence and a good dose of educated realism that normalizes the many possible outcomes of birth for women and their families, babies and bodies. Thus, the re-centering of the maternal subjective in homebirth videos online, and the simultaneous romanticization and desensitization that goes along with it, offers a complex visual culture of birth.

If, as Michelle Citron argues in her article, "Fleeing from Documentary: Autobiographical Film/Video and the 'Ethics of Responsibility'" that "autobiographical films and videos bear witness to our lives" $(1999,2715)$, the question raised here is what contemporary amateur representations of homebirth on-line might tell us about the personal and political struggles of (home)birthing women and their families, and to what extent such documents contribute to and expand upon the feminist (re)visioning of pregnancy and childbirth in the tradition of experimental homebirth films. Like experimental filmmaker Stan Brakhage's unprecedented depictions of childbirth in and out of the domestic sphere (beginning with his first childbirth film Window Water Baby Moving in 1959), are these representations of the maternal body on-line crossing a new frontier of the taboo? Figures like Shanley whose unassisted birthing videos have been either flagged as inappropriate by YouTube users or in some cases deleted by YouTube would certainly say so, especially given the prevalence and acceptance of invasive medical images and procedures performed on birthing women's 
bodies not only on the Internet but in our culture at large. Thus, images of amateur homebirth advocacy on-line constitute a continuation of what appears to be an important "counterhistory" of birth and embodiment in our visual culture.

However, on-line images of homebirth do not necessarily succeed in disrupting normative seeing practices, at least not in the ways that avant-garde artists have historically attempted, theorized and fought for. In fact, the format itself and the visual trends that dominate the bulk of home movie imagery on-line arguably cement images of the procreative body into what has now become the "normative" visual landscape of the Internet, varied as it may be. Rooted in the spectacle of what was formerly viewed as mundane or private, the Internet's embrace of the (home)birthing body as a widespread visual subject relies on an exploitation of the very notion of visibility itself - and, I would argue, at the cost of ownership. Here I am not referring to ownership of the birth experience or even one's body - although these are two important elements of agency that birthing women can and have gained both in and out of the hospital - but ownership of the images and stories that accompany an otherwise empowered birth.

Certainly, the videos I have referred to here achieve the goal of regaining ownership of the maternal birthing body in ways that does not pathologize it, yet in the efforts to publicize (and thus normalize) the experiences of happy homebirthers, these images and stories enter the public domain in such a way that generally revokes practically any ownership over them at all. For instance, while most of the videos on birthvideos.tk forefront each video as "used with permission" (along with the "stamp" of the website evident throughout the video), instructions on how to download or embed the video onto your computer for personal use are also explicit. It appears that ownership and agency is demonstrated in these images through the fight for an autonomous birth experience and the ability to document (and publicize) these images, and that in order for these images to effectively enter the politicized public domain, the strings that link these images as belonging to specific individuals may be effectively broken. That is not to say that these images lose their political relevance, but it does posit a specific challenge of sorts to the "personal is political" stance that may motivate women and their families to sacrifice ownership of their birthing images to begin with.

And yet, the bulk of on-line (home)birth videos powerfully demonstrates two core "messages" about procreation: on the one hand, birth is incredibly diverse, with a rich diversity of location, birthing positions, sounds, company, and methods - from gay and lesbian birthing experiences, hypnobirthing, waterbirth, unassisted birth, or lotus birth (in which the placenta remains connected to the newborn until the umbilical cord falls off on its own), the approaches to and experiences of childbirth are unmistakably vast. On the other hand, birth is depicted as relatively universal - a mother (or, in intervention-based birth practices, a doctor) delivers a baby. It is precisely within this tension between diversity and universality that homebirth images on the Internet are both politically and personally invested while at the same eerily apolitical and perhaps even average - particularly in the realm of what has become normative seeing and viewing practices on-line where the spectacle of the would-be miraculous and the so-called mundane exist on the same platform. This multiplicity makes the Internet both a democratic and potentially political space while simultaneously serving as a site for a desensitizing of-sorts of the spectacle, effectively translating the previously taboo or pornographic back into the realm of the 
(publicized) mundane. Thus, the video realm of the Internet is particularly seductive for homebirth advocates as a consciousness-raising tool that has the capacity to visually normalize the "natural" (i.e., the non-intervened) maternal and birthing bodies, although an accompanying agenda for action, like that modeled by the International Cesarean Awareness Network, is necessary for medical and legislative changes to occur (since images in and of themselves are not enough to undo the pathologization of birth that has been cemented into mainstream approaches and practices surrounding the maternal body).

Indeed, the many rich and diverse representations of amateur homebirth home movies on the Internet is part of an on-going visual effort to make and exhibit visual documents of and about the maternal, birthing body that upholds the (w)holistic model of childbirth, thus furthering the emotional, visual, and political stakes of the homebirth movement both on-line and in real life. Depictions of homebirth are certainly rooted in their spirit of collaboration, the need for personal and political visual commentary, and a deep consideration and celebration of birthing women and their families. As homebirth advocacy gains new and even stronger grounds, the synonymous acts of giving birth and picking up the film/video camera will continue to empower women and their families both in practice and in vision.

\section{Notes}

1. URL addresses ending in "Dot TK" indicate the Internet country code for Tokelau (top level domain) of New Zealand, which provides free domain names for any interested party so long as the website is visited consistently. (www.dot.tk/en/index.html)

2. Kelly Milotay's article on Cephalopelvic Disproportion (CPD) on The International Cesarean Awareness Network (ICAN) outlines CPD myths and offers suggestions that may lower women's risk of such a diagnosis, including prenatal chiropractic care, movement in labor, and the use of a doula. Milotay's article links to ICAN's "Question CPD" video by Elaine Mills on YouTube (ICANvoices channel: November 16, 2007, youtube.com/watch? $v=$ roFVkDV45MM) whose slideshow features previously Cesareanoperated mothers successfully giving birth vaginally to babies that are in fact bigger than those subsequently birthed, as well as to Minnesota midwife Gail Tulley's spinningbabies.com, a website that offers steps to optimal fetal positioning based on the principles of balance, gravity and movement. (ican-online.org/vbac/cephalopelvicdisproportion-cpd)

3. For a discussion on depictions of the pregnant subject by the maternity clothing industry, see Sandra Matthews and Laura Wexler's chapter "Promoting Pregnancy: Instruction, Advertising and Public Policy" in their book Pregnant Pictures (New York: Routledge, 2000).

4. Both GoldenPalace.com, an internet casino based in the Mohawk Territory of Kahnawake, Canada, and Globat, a web-hosting company, have won bids to document the birth and use images of the pregnant subject (whose abdomen bares a temporary tattoo of the company's logo) on their websites. For instance, Elise Harp's birth and preand post-birth interviews can be found on YouTube (youtube.com/watch?v=IgsufJCliM0)

5. Citron, Michelle. "Fleeing from Documentary: Autobiographical Film / Video and the 'Ethics of Responsibility." Feminism and Documentary. Eds. Diane Waldman and Janet Walker. Minneapolis, Minnesota: University of Minnesota Press, 1999. 267-270 (chapter 12).

Shira Segal is Ph.D. Candidate and Associate Instructor, Department of Communication and Culture, Indiana University. Email: sbsegal@indiana.edu 